\title{
Construction of the Small Intestine Epithelial Cell Model Based on Molecular Dynamics Simulation and Preliminary Exploration of Drug Intestinal Absorption Prediction
}

\section{Yanshuang Shi}

Beijing University of Chinese Medicine

Menke Sheng

Beijing University of Chinese Medicine

Qingsong Qu

Beijing University of Chinese Medicine

Yuyao Liao

Beijing University of Chinese Medicine

Lijng Lv

Beijing University of Chinese Medicine

Jiaqi Yang

Beijing University of Chinese Medicine

Zhixun Li

Beijing University of Chinese Medicine

Qing Zhou

Beijing University of Chinese Medicine

XingXing Dai

Beijing University of Chinese Medicine

Xinyuan Shi ( $\nabla$ shixinyuan01@163.com)

Beijing University of Chinese Medicine

\section{Research Article}

Keywords: Small intestinal epithelial cell model, Intestinal absorption prediction, Parameter evaluation method, Molecular dynamics simulation

Posted Date: July 15th, 2021

DOI: https://doi.org/10.21203/rs.3.rs-683560/v1 
License: (c) (i) This work is licensed under a Creative Commons Attribution 4.0 International License. Read Full License 


\section{Abstract}

In this study, molecular dynamics simulation was applied to the construction of small intestinal epithelial cell membrane and prediction of drug absorption. First, we constructed a system of a small intestinal epithelial cell membrane that was close to the real proportion and investigated the effects of temperature, water layer thickness, and ionic strength on membrane properties to optimize environmental parameters. Next, three drugs with different absorptivity, including Ephedrine (EPH), Quercetin (QUE), and Baicalin (BAI), were selected as model drugs to study the ability of drugs through the membrane by the free diffusion and umbrella sampling simulation, and the drug permeation ability was characterized by the free diffusion coefficient $D$ and free energy barrier $(\triangle G)$ in the processes. The results showed that the free diffusion coefficient $D^{\prime}$ and $\triangle G^{\prime}$ orders of the three drugs were consistent with the classical experimental absorption order, indicating that these two parameters could be used to jointly characterize the membrane permeability of the drugs.

\section{Introduction}

Oral drug administration is the most common and convenient method for clinical administration, while the small intestine is the main place for oral drug absorption. Most drugs need to pass through the cell membrane of small intestinal cells and then reach the systemic blood circulation through passive diffusion or carrier-mediated active transport. Therefore, predicting the intestinal absorption of oral drugs is of great significance in evaluating the therapeutic effect of drugs [1].

The most effective way to evaluate the intestinal drug absorption is through the intestinal absorption model, which is currently commonly used includes cell model, animal model, and artificial biofilm model. Caco-2 cell model, the most commonly used cell model, is widely used because it is close to the absorption environment of the human body [2-4]. Considering that there are other transport carriers in Caco-2 cells, such as peptides, organic cation transporters, organic anion transporters, etc., it may lead to a lower level of drug absorption than the actual absorption level [5]. As for the animal model, in vivo and in situ animal models have the disadvantages of high cost, long period and complex processing of biological samples, and in vitro animal models have problems with poor accuracy of experimental data, which make experimental animal models not suitable for early drug intestinal absorption research, although they can best reflect the actual absorption of drugs [6-7]. With the advantages of high throughput, low cost, and high flexibility, the parallel artificial membrane infiltration model is an ideal in vitro model for preliminary drug absorption prediction through biofilm [8]. However, the phospholipids in this model are weakly bound together by intermolecular interactions, making them fluid, deformed, misplaced, and difficult to manipulate [9]. Therefore, the traditional drug absorption model is not suitable for the high-throughput screening of early drug intestinal absorption studies, and an efficient and simple model is urgently needed to evaluate drug absorption.

Molecular dynamics (MD) simulations, a new technology developed in recent years, expounds the mechanism of drug and biofilm action from micro and mesoscopic scales to obtain important 
information that is difficult to obtain in the experiment, so it is widely used in drug absorption [1012]. Mojumdar etc.[13] applied it to the study of transmembrane absorption of articaine, a local anesthetic, and concluded that the effect of drug molecules on membrane electrostatic potential might be one of the molecular mechanisms of anesthetic effect. Boggara etc. [14], Simulated the diffusion process of aspirin and ibuprofen in DPPC membrane and compared the influence of drugs with different charged forms on membrane permeability under the influence of $\mathrm{pH}$. Thus, it was concluded that the electrically neutral drug molecules passed through the phospholipid membrane through passive diffusion, while the electrically charged drug molecules might pass through the transient pore effect. The results above show that MD simulations study the effect of drugs on biofilm structure and the process of drug absorption through the membrane by establishing a cell membrane model from the point of intermolecular interaction. Therefore, it provides a new method for predicting intestinal drug absorption and the study of the interaction law between drugs and biofilm.

In this paper, the small intestinal epithelial cell membrane model was built based on the CHARMM-GUI platform. The effects of temperature, water layer thickness, and ionic strength on the model were investigated through MD simulation so as to optimize the parameters of the established model and make it closer to the real small intestinal epithelial cell membrane. In addition, the free diffusion and umbrella sampling processes of drugs with different absorption rates were simulated, and the parameters in the simulated membrane permeation process were used as evaluation indexes. By comparing the simulation results with the experimental results, the reliability of the model was proved, and the parameter evaluation method of intestinal drug absorption matching with the model was established in order to realize the rapid and simple prediction of intestinal absorption.

\section{Method}

\subsection{Establishment of small intestinal epithelial cell model}

Small intestinal epithelial biofilms can be divided into Apicals (AP) facing the intestinal lumen and Basolateralside $(\mathrm{BL})$ facing the blood. The lipids on both sides of the membrane are mainly composed of phospholipid, glycosolipid, and cholesterol, and the ratio of the three in the AP is 1:1:1, while the ratio of the three in the $\mathrm{BL}$ is 5:3:2 [15].In the composition of the lipid membrane, the phospholipids involved include phosphatidylcholine (POPC), phosphatidylethanolamine (POPE), phosphatidylinositol (POPI), phosphatidylserine (POPS), and glycosipids include glycosphingolipids (GMI). All of these molecules have been verified by coarsening on the martini official website. The Coarse Grain (CG) models are shown in Fig. 1.

This research was based on Gromacs 5.0.7, and the self-assembly of lipid bilayer was established by using CHARMM-GUI. According to the proportion of AP and BL membranes, about 600 lipid molecules were placed in a $14 * 14 * 8 \mathrm{~nm}$ box, and water molecules and a certain molar concentration of $\mathrm{Na}^{+}$and $\mathrm{Cl}^{-}$were added, respectively [16-17]. 


\subsection{Optimization of model parameters}

The performance of the small intestinal epithelial cell membrane was affected by temperature [18-19], water layer thickness, and ionic strength [20-21], respectively. The properties of the mixed membrane were studied by observing the changes of the above three factors so as to optimize the environmental parameters and establish the small intestinal epithelial cell membrane model close to the real composition. In this way, we can better predict the intestinal absorption of the drug. In this paper, Area per lipid (APL), Bilayer thickness, and diffusion coefficient $D$ were selected as evaluation parameters to screen out the optimal model parameters.

The annealing simulation was used to study the phase transformation process of the mixed membrane, to investigate the influence of temperature on the membrane properties. The annealing simulation time was $1.6 \mu \mathrm{s}$, and the whole temperature span was $160 \mathrm{~K}(230-390 \mathrm{~K})$. For a bilayer system composed of only lipid molecules, the water layer thickness was generally $22.5 \AA$, and the ionic strength was $0.15 \mathrm{~mol}$. At this time, the lipid membrane can maintain the equilibrium state of water and ions [22]. However, the bilayer system constructed in this paper was composed of multiple lipid molecules. In order to screen out the optimal environmental parameters to meet the morphological requirements and equilibrium state of the lipid membrane, the effects of different water layer thickness and ionic strength were selected to investigate the established model. The water layer thickness was $10.5,22.5$, and $3345 \AA$, and the ionic strength was $0.05,0.15$, and $0.25 \mathrm{~mol}$, respectively.

\subsection{Predictive characterization of drug absorption through the membrane}

In this paper, three drugs, EPH, QUE, and BAl, previously studied by our research group, were selected as model drugs. The absorptivity experimental data of each drug was shown in Table 1, where -LogP was obtained by converting the apparent permeability coefficient (Papp) in the Caco-2 model. The lower the LogP of a drug, the better the drug was absorbed, so the cell experiment results showed that the order of absorption of the three drugs was FER $\otimes$ QUE $\triangle B A I$.

In the optimized small intestinal epithelial cell membrane system, we simulated the free diffusion of three model drugs into the cell membrane and the process of umbrella sampling through the cell membrane and selected two parameters, diffusion coefficient $D$ and $\triangle G$, to characterize the free diffusion rate and membrane permeability of drugs. By comparing the simulation results with the cell experimental results, the reliability of the small intestinal epithelial cell model was verified, and the parameter evaluation method for the prediction of oral drug absorption through the membrane was established at the simulation level.

To simulate the free diffusion of drugs into the cell membrane, drug molecules with a certain molar concentration were first placed in the water layer of the cell membrane to replace the corresponding number of water molecules (Fig. 2). In order to simulate the process of drug molecules through the 
membrane, a drug molecule was placed in the center of the double lipid membrane, and the double lipid membrane model and each drug molecule were located in the origin coordinate $(X=0, Y=0, Z=0)$, and then by combining their atomic coordinates to form a new multi-component complex. Using umbrella sampling to apply a traction force to the drug molecule makes it pass through the lipophilic center and hydrophilic center of the lipid bilayer along the Z-axis until it reached the water layer outside the membrane so as to obtain the complete trajectory of the drug molecule passing through the membrane [23].

\subsection{Evaluation parameters}

APL, the area of a single lipid molecule in the lipid bilayer cross-section, can be used to illustrate the density of the lateral arrangement of small intestinal epithelial cell membrane molecules in the system and evaluate whether the system is in equilibrium according to its change over time. APL can be calculated from gmx energy in GROMACS.

Bilayer thickness, the thickness of lipid bilayer of the cell membrane, can be used to characterize the longitudinal stacking of cell membrane molecules in the small intestine epithelium. gmx density in GROMACS can be used to obtain the density of the lipid head group, and the bilayer thickness is obtained by calculating the distance of the peak in the mass distribution map of the lipid head group along the Zaxis.

Mean square displacement(MSD), the square of the distance from the initial position of the particle at $t$ time. According to the curve of the MSD with time, the particle diffusion coefficient $D$ can be obtained. For the lipid tail chain, the transverse diffusion coefficient $D$ can characterize the fluidity of the tail chain, while for the drug molecule, the Z-axis diffusion coefficient $D$ can characterize the rate of its entry into the lipid bilayer. The diffusion coefficient $\mathrm{D}$ of drugs and phospholipid molecules can be calculated by $g m x$ msd in GROMACS.

$\triangle G$, the free energy change of drugs passing through biofilms, can investigate the difficulty of different drugs passing through biofilms, and the smaller the $\triangle G$ is, the easier the drug is to penetrate the membrane. gmx wham in GROMACS can be used to transform the probability distribution of drugs in lipid membrane to obtain the free potential energy surface, through which the $\triangle G$ in the membrane penetration process of each drug can be obtained.

\section{Results And Discussion}

\subsection{The results of model parameter optimization}

The small intestinal epithelial cell membrane model preliminarily established in this paper is shown in Fig.3. In order to better predict the intestinal absorption of drugs, the influence of temperature, water layer thickness, and ionic strength on the small intestinal epithelial cell model was investigated in this paper to select the optimal environmental parameters. 


\subsubsection{Effects of temperature on membrane properties}

The changes of APL of AP and BL with temperature are shown in Fig.4 and Fig. 5. It can be seen from the figure that in the temperature range of 230-390 K, the influence of temperature on both sides of the membrane is roughly the same, and the APL increases with the increase of temperature. For the heating process, there are two sharp points ( Fig.4a and Fig.5a). A smaller mutation point is between 280-290K, before which APL is small and almost unchanged, indicating that the lipid head groups are well arranged and tightly packed, and the membrane is in a complete gel state. While APL increased significantly after this mutation point, indicating that the arrangement of lipid head groups was looser than before, which was speculated to be related to the pre-phase transition of the cell membrane. The other mutation point was between $310-330 \mathrm{~K}$. After this mutation point, APL increased rapidly and did not appear saturation point with the increase of temperature. This indicates that with the increase of temperature, the thermal energy destroys the interaction between the lipid head groups, which increases the free space of the lipid head groups, leading to the complete transition to the liquid state. For the cooling process, APL decreases uniformly with the decrease of temperature, and there is no obvious mutation point on both sides of the membrane with the decrease of temperature. ( Fig.4b and Fig.5b).

The membrane thickness of AP and BL varies with temperature, as shown in Fig. 6 . In the temperature range of 230-390 K, the influence of temperature on both sides of the membrane is roughly the same, and the membrane thickness decreases with the increase of temperature. Moreover, for the heating process, there are corresponding turning points around the two temperatures at which the phase transition occurs, which is consistent with the results of APL. For the cooling process, there is no obvious turning point except that the AP film has a big fluctuation at $250 \mathrm{~K}$, which is also roughly consistent with the results of APL without mutation during the cooling process. In addition, to further explain the influence of temperature on the membrane morphology, AP membrane was taken as an example to analyze the lipid molecular arrangement at three temperature points where the thickness of the membrane was significantly different during the heating process (Fig. 7). The results show that the higher the temperature is, the wider the peak shape of the density distribution map of the lipid head-based beads along the Z-axis is, which indicates that the higher the temperature, the more disordered the lipid molecules are arranged. On the contrary, the lower the temperature, the narrower the peak shape of the density distribution map along the Z-axis of the lipid head-based beads, indicating the more orderly the arrangement and the larger the peak distance between the two peaks, indicating the larger the film thickness. This is also consistent with the results of changes in APL. The increase of temperature would destroy the interaction force between the lipid head-based beads, making them move more freely and arrange more disorderly.

The morphology of AP and BL varies with temperature, as shown in Fig. 8. As can be seen from the figure, at $250 \mathrm{~K}$ and $280 \mathrm{~K}$, the lipid membrane is tightly arranged and orderly, and the membrane thickness is basically unchanged, so the membrane is in a complete gel state at this time. At $310 \mathrm{~K}$, the arrangement of beads was obviously looser than before, indicating that a pre-phase transition occurred at some time point between $280-310 \mathrm{~K}$, and the film had already transited from gel state to liquid state, but the film still 
maintained a good film shape without severe deformation. When the temperature reached $340 \mathrm{~K}$, the lipid bilayer began to bend and deform, and with the increase of temperature, the film morphology became more curly until the deformation was severe and no longer existed in the form of the bilayer, indicating that a phase transition occurred at some time point between $310 \mathrm{~K}-340 \mathrm{~K}$. The above changes in membrane morphology with temperature more intuitively prove the previous results of APL and membrane thickness.

The above results indicated that the pre-phase transformation of the small intestine epithelial cell model was between $280 \mathrm{~K}$ and $290 \mathrm{~K}$, and the phase transformation was between $310 \mathrm{~K}$ and $330 \mathrm{~K}$, which was consistent with the reported cell membrane phase transformation temperature near the physiological temperature[24-26]. Moreover, it is worth noting that when the temperature is maintained between $290 \mathrm{~K}$ and $310 \mathrm{~K}$, the gelatinous state of the membrane coexists with the liquid state, and the lipid bilayer of the membrane is in good shape without serious bending deformation, which is close to the real state of the membrane.

\subsubsection{Effects of water layer thickness and ionic strength on membrane properties}

The results of APL and membrane thickness changing with water layer thickness and ionic strength are shown in Fig. 9. When the thickness of the water layer is $22.5 \AA, A P L$ is $0.46 \mathrm{~nm}^{2}$, and membrane thickness is $4.1 \mathrm{~nm}$. With the thickening of the water layer, the APL increases, and the membrane thickness also becomes thicker. This indicates that increased hydration causes more water molecules to contact with the lipid head region, which reduces the compactness of lipid molecules horizontally, thus increasing APL, while the entry of excess water molecules also increases the membrane thickness when the ionic strength increases or decreases, the changes of APL and membrane thickness tend to be stable, indicating that the ionic strength has little effect on the membrane morphology in the model built in this paper.

In conclusion, when the temperature is maintained between $290 \mathrm{~K}$ and $310 \mathrm{~K}$, the APL of the membrane constructed in this paper is stable at about 0.46-0.47 nm. At this time, the lipid bilayer of the membrane is in good shape, and the membrane is neither in absolute gel state nor will there be severe bending deformation, but close to the real membrane state, which will be conducive to the follow-up study of drug permeation membrane absorption. Therefore, the simulated temperature we selected was $310 \mathrm{~K}$, which was also consistent with the temperature of the cell membrane under physiological state. The water layer thickness was $22.5 \AA$, and the ionic strength was $0.15 \mathrm{~mol}$, which was consistent with the relevant literature reports.

\subsection{Predictive characterization of drug absorption through the membrane}

In this section, three model drugs QUE, EPH, and BAl, were used to simulate the complete transmembrane process of the drug by free diffusion and umbrella sampling in the optimized model. 
Free diffusion simulation, in which the drugs enter the cell membrane through free diffusion, can be used to reflect the difficulty of drug penetration preliminarily. Considering the interaction and absorption process between drugs and cell membranes in the physiological environment, the longitudinal drug diffusion along the Z-axis is closer to the real drug permeation process. While the transverse drug diffusion along the X-axis mainly indicates that the drug has different degrees of disturbance to the lateral distribution of lipid molecules. Therefore, the MSD and diffusion coefficient $\mathrm{D}$ of the drug along the Z-axis were calculated respectively to characterize the free diffusion velocity of the drug, and the results were shown in Fig.10 and 11. According to the figures, although the proportion of lipid molecules on both sides of the membrane was different, the longitudinal diffusion coefficient $D$ of each model drug also had some differences, but the overall diffusion trend remained the same, and the diffusion coefficient D was ordered as EPH > QUE > BAl. The results showed that the changes of lipid membrane components in the selected lipid components in this study had little effect on the drug diffusion rate, and the order of three drugs permeating the membrane reflected by free diffusion simulation was EPH > QUE > BAl, which was consistent with the results of cell experiment.

Umbrella sampling simulation, in which the drug passes through the intact cell membrane under the action of the traction force, can be used to reflect the drug penetration capacity. In the simulation, the free energy potential surface(Fig. 12) of each model drug through the membrane was obtained, and $\triangle \mathrm{G}$ of each model drug through the membrane was calculated by using PMF graph data (Table 2).

It can be seen from the table that the $\triangle \mathrm{G}$ of each model drug through the membrane of both sides is different to some extent, but the same trend on both membranes of the $\triangle G$ reflected the same tendency of membrane permeability of each model drug, indicating that the change of lipid membrane composition in the selected lipid components in this study had little effect on the membrane permeability of the drug. In addition, it can also be seen from the table that the $\triangle \mathrm{G}$ of each model drug is greatly different, and the descending order is EPH, QUE, and BAl, indicating that the membrane permeability of these three model drugs is $\mathrm{EPH}>\mathrm{QUE}>\mathrm{BAl}$, which is also consistent with the experimental absorption order.

In this part, the free diffusion simulation, which preliminarily characterized the difficulty of drug membrane penetration, combined with the umbrella sampling simulation, which evaluated the ability of drug membrane penetration, was used to reflect the ability of drug absorption. The results showed that the free diffusion coefficient $D$ and $\triangle G$ order of the three drugs in two simulations were consistent, and the order was EPH > QUE > BAl, which is also consistent with the order of absorption in cell experiments. This indicates that the model established in this paper is reliable, and the two parameters can be used to reflect the absorption rate of drugs to a certain extent jointly.

\section{Conclusion}

In this study, we have built a model of the small intestinal epithelial cell membrane that is close to the real proportion and investigated the influence of temperature, water layer thickness, and other factors on 
membrane properties to optimize environmental parameters. The results show that when the simulated temperature is $310 \mathrm{~K}$, the water layer thickness is $22.5 \AA$, and the ionic strength is $0.15 \mathrm{~mol}$, the membrane is in good condition, which is close to the real cell state.

We also simulated the process of free diffusion and umbrella sampling of different drug models and characterized the drug permeation ability by the changes of parameters during the process of drug molecules permeating the membrane. The results show that the free diffusion coefficient $D$ and free energy barrier of the model drugs reflect the same tendency as the classical experimental results, which can jointly characterize the absorption rate of the drug.

The focus of our work is to build a model of small intestinal epithelial cells close to the human body and to establish a parameter evaluation method for the prediction of oral drug permeable membrane absorption at the simulation level. Compared with the traditional intestinal absorption model, this model has the advantages of saving time, resources and manpower, and is suitable for high throughput screening for early studies of intestinal drug absorption. Future work will focus on the study of the drug permeation mechanism

\section{Declarations}

\section{Funding}

This work was funded by the National Natural Science Foundation of China (Grant No.81473364).

\section{Conflicts of interest/Competing interests}

The authors declare that there is no conflict of interests regarding the publication of this article.

\section{Availability of data and material}

The datasets used or analyzed during the current study are available from the corresponding author on reasonable request.

\section{Code availability}

The experiment was carried out on the basis of Gromacs 5.0.7, Packmol 18.013, Visual Molecular Dynamics 1.9.2, and CHARMM-GUI.

\section{Authors' contributions}

YS and XS contributed to the conception of the study; YS, MS and XD performed the experiment; QQ, YL, and LL participated in the data collection and the analysis of the results; $Y S$ and $X S$ aided in drafting and revising the manuscript; JY, ZL, and QZ helped perform the analysis with constructive discussions.

\section{Consent to participate}


Using the small intestinal epithelial cell membrane model established in this study and three representative drugs as models, the simulated force field was fully validated.

\section{Consent for publication}

This study provides a model of the small intestinal epithelial cells close to the true composition and a new method for predicting intestinal drug absorption.

\section{Acknowledgments}

First of all, I would like to take this opportunity to express my heartfelt thanks to my supervisor, Ms. Shi, who gave me a lot of useful suggestions on topic selection, thinking, and writing with her professional knowledge. Secondly, I would like to thank my partners for their efforts in data collection, experimental operation, and data processing. Without their help, it would be more difficult for me to complete my study and this paper. Last but not least, I would like to thank those leaders, teachers, and working staff at Beijing University of Chinese Medicine for their convenience in terms of experimental equipment and resources, which contributed to the completion of this article.

\section{References}

1. Liu JY, Lee KF, Sze CW, Tong Y, Tang CW, Ng TB, Zhang YB (2013) Intestinal absorption and bioavailability of traditional Chinese medicines $\triangle a$ review of recent experimental progress and implication for quality control. J Pharm Pharmacol 65(5). https://doi.org/10.1111/j.20427158.2012.01608.x

2. Yang H, Zhai BT, Fan Y, Wang J, Sun J, Shi YJ, Guo DY (2018) Intestinal absorption mechanisms of araloside $\mathrm{A}$ in situ single-pass intestinal perfusion and in vitro Caco-2cell model. Biomed Pharmacother 106:1563-1569. https://doi.org/10.1016/j.biopha.2018.07.117

3. Willenberg I, Michael M, Wonik J, Bartel LC, Empl MT, Schebb NH (2015) Investigation of the absorption of resveratrol oligomers in the Caco-2cellular model of intestinal absorption. Food Chem167(jan.15):245-250. https://doi.org/10.1016/j.foodchem.2014.06.103

4. Liu W, Pan H, Zhang CY, Zhao LL, Zhao RX, Zhu YT, Pan WS (2016) Developments in Methods for Measuring the Intestinal Absorption of Nanoparticle-Bound Drugs. Int J Mol Sci 17 (7);1171. https://doi.org/10.3390/ijms17071171.

5. Bohets H, Annaert P, Mannens G, Beijsterveldt LV, Anciaux K, Verboven P, Meuldermans W, Lavrijsen K (2001) Strategies for absorption screening in drug discovery and development. Curr Top Med Chem, 1(5):367-383. https://doi.org/info:doi/10.2174/1568026013394886

6. Zhang ZC, Li XX, Li LL (2015) Application of Animal Intestinal Absorption Model in Drug Development. Medical Recapitulate 21(05):775-778. https://doi.org/10.3969/j.issn.10062084.2015.05.003. 
7. Zhang ZC, Li XX, Li LL (2015) Application of Animal Intestinal Absorption Model in Drug Development. Medical Recapitulate 21(05):775-778. https://doi.org/10.3969/j.issn.10062084.2015.05.003.

8. Wu YF, Liu H, Ni JM (2011) Advances in parallel artificial membrane permeability assay and its applications. Acta Pharmacol Sin,46(08):890-895. https://doi.org/10.16438/j.05134870.2011.08.012

9. Avdeef A (2005) The rise of PAMPA. Expert Opin Drug Metab Toxicol 1(2) $₫ 325-342$. https://doi.org/10.1517/17425255.1.2.325

10. Dave V, Yadav RB, Yadav S, Sharma S, Sahu RK, Ajayi AF (2018) A critique of computer simulation software's used in pharmacokinetics and pharmacodynamics analysis. Current Clinical Pharmacology 201813 (4):216-235. https://doi.org/10.2174/15748884713666181025144845

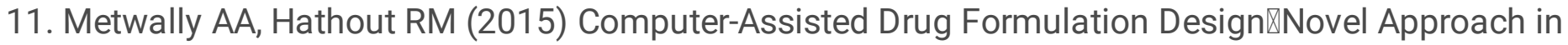
Drug Delivery. Mol Pharm,12(8):2800-2810. https://doi.org/10.1021/mp500740d

12. Wu ZM, Ding HO, Yang C, Guo SJ, Dai XX, Shi XY (2018) Dissipative Particle Dynamics Study on Platycodin's Solubilization Enhancing Effect Towards Five Drug Components. Chem Res Chinese U, 35(1). https://doi.org/10.1007/s40242-018-8250-7

13. Mojumdar EH, Lyubartsev AP (2010) Molecular dynamics simulations of local anesthetic articaine in a lipid bilayer. Biophys Chem 153(1): 27-35. https://doi.org/10.1016/j.bpc.2010.10.001

14. Boggara MB, Krishnamoorti R (2010) Partitioning of nonsteroidal antiinflammatory drugs in lipid membranes: a molecular dynamics simulation study. Biophys J 98(4):586-595. https://doi.org/10.1016/j.bpj.2009.10.046

15. Simons K, Meer GV (1988) Lipid sorting in epithelial cells. J Cell Biochem 36(17):6197-202. https://doi.org/10.1021/bi00417a001

16. Jo S, Klauda JB, Im W (2009) CHARMM-GUI Membrane Builder for Mixed Bilayers and Its Application to Yeast Membranes. Biophys J, 96(Supplement 1):41a. https://doi.org/10.1016/j.bpj.2009.04.013

17. Qi Y, Ingólfsson HI, Lee J, Im W (2015) CHARMM-GUI Martini Maker for Coarse

18. Grained Simulations with the Martini Force Field. Biophys J 108(2); 161 a. https://doi.org/10.1021/acs.jctc.5b00513

19. Toplak H, Batchiulis V, Hermetter A, Hunziker T, Honegger UE, Wiesmann UN (1990) Effects of culture and incubation conditions on membrane fluidity in monolayers of cultured cells measured as fluorescence anisotropy using trimethylammoniumdiphenylhexatriene (TMA-DPH). Biochem Biophys Acta 1028(1):67-72. https://doi.org/10.1016/0005-2736(90)90266-Q

20. Zeljko S, Hermann T, Roger Z, Honegger UE, Bhlmann R, Wiesmann UN (1992) Cultured human skin fibroblasts modify their plasma membrane lipid composition and fluidity according to growth temperature suggesting homeoviscous adaptation at hypothermic (30 degrees $\mathrm{C}$ ) but not at hyperthermic (40 degrees $\mathrm{C}$ ) temperatures. Biochem Biophys Acta 1104(1):31-37. https://doi.org/10.1016/0005-2736(92)90128-9 
21. Sun L, Mao JS, Zhao Y, Quan CS, Zhong ML, Fan SD (2017) Coarse-grained molecular dynamics simulation of interactions between cyclic lipopeptide Bacillomycin D and cell membranes. Molecular Simulation 44(2):1-13.https://doi.org/10.1080/08927022.2017.1384632

22. Kolds $\varnothing$ H, Shorthouse D, He'lie J, Sansom MSP (2014) Lipid Clustering Correlates with Membrane Curvature as Revealed by Molecular Simulations of Complex Lipid Bilayers. PLoS Comput Biol 10(10):e1003911. https://doi.org/10.1371/journal.pcbi.1003911

23. Yang C, Dai XX, Yang SF, Ma LN, Chen LP, Gao RL, Wu XW, Shi XY (2019) Coarse-Grained Molecular Dynamics Simulations of the Effect of Edge Activators on the Skin Permeation Behavior of Transfersomes. Colloid Surface B, 183:110462. https://doi.org/10.1016/j.colsurfb.2019.110462

24. Bao JZ, Davis CC, Schmukler RE, (1991) Impedance Spectroscopy of Human Erythrocytes: Constant Phase Angle Characteristics and a Membrane Phase Transition. Biophys J 61(5): 1427-1434. https://doi.org/10.1016/s0006-3495(92)81948-3

25. Bao JZ, Davis CC, Schmukler RE (1992) Frequency domain impedance measurements of erythrocytes. Constant phase angle impedance characteristics and a phase transition. Biophys J 61(5):1427-1434. https://doi.org/10.1016/S0006-3495(92)81948-3

26. Weber $P$, Wagner M, Strauss WSL, Schneckenburger H (2007) Fluorescence imaging of cholesterol and temperature dependent cell membrane dynamics. SPIE-OSA Biomedical Optics 6633. https://doi.org10.1117/12.728136

\section{Tables}

Table 1. Experimental -LogP of model drug

\begin{tabular}{|ll|}
\hline DRUG & $-\operatorname{LogP}$ \\
\hline EPH & 4.610 \\
QUE & 5.638 \\
BAI & 5.783 \\
\hline
\end{tabular}

Table 2. Free energy barrier $\mathrm{G}$ of the permeable membrane of each drug

\begin{tabular}{lll} 
DRUG & \multicolumn{2}{c}{$\triangle \mathrm{G}(\mathrm{kJ} / \mathrm{mol})$} \\
\cline { 2 - 3 } & AP & $\mathrm{BL}$ \\
\hline EPH & 30.80 & 25.45 \\
\hline QUE & 36.29 & 29.95 \\
\hline BAI & 41.85 & 43.96
\end{tabular}


Figures
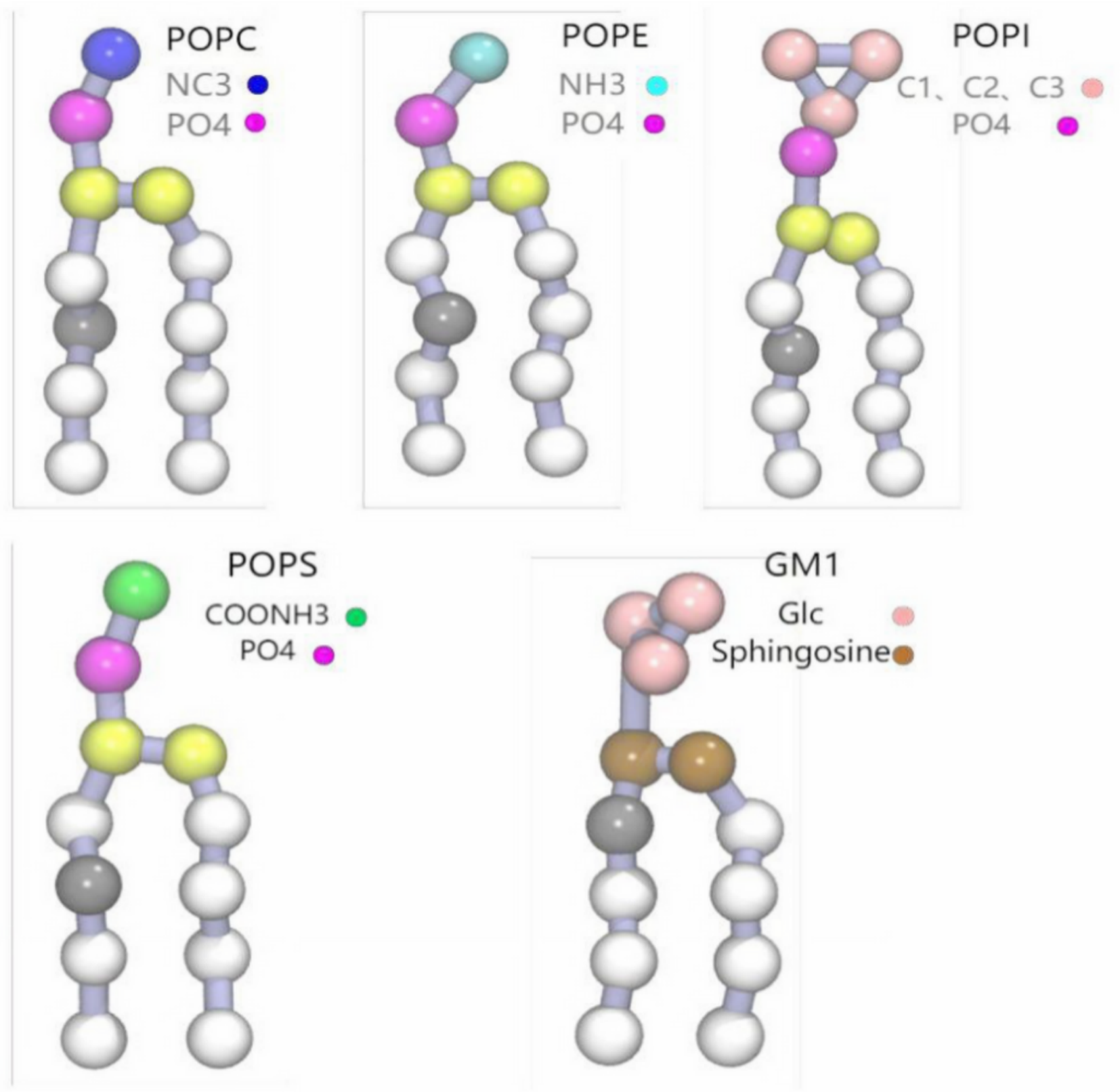

Figure 1

CG model of lipid molecules 


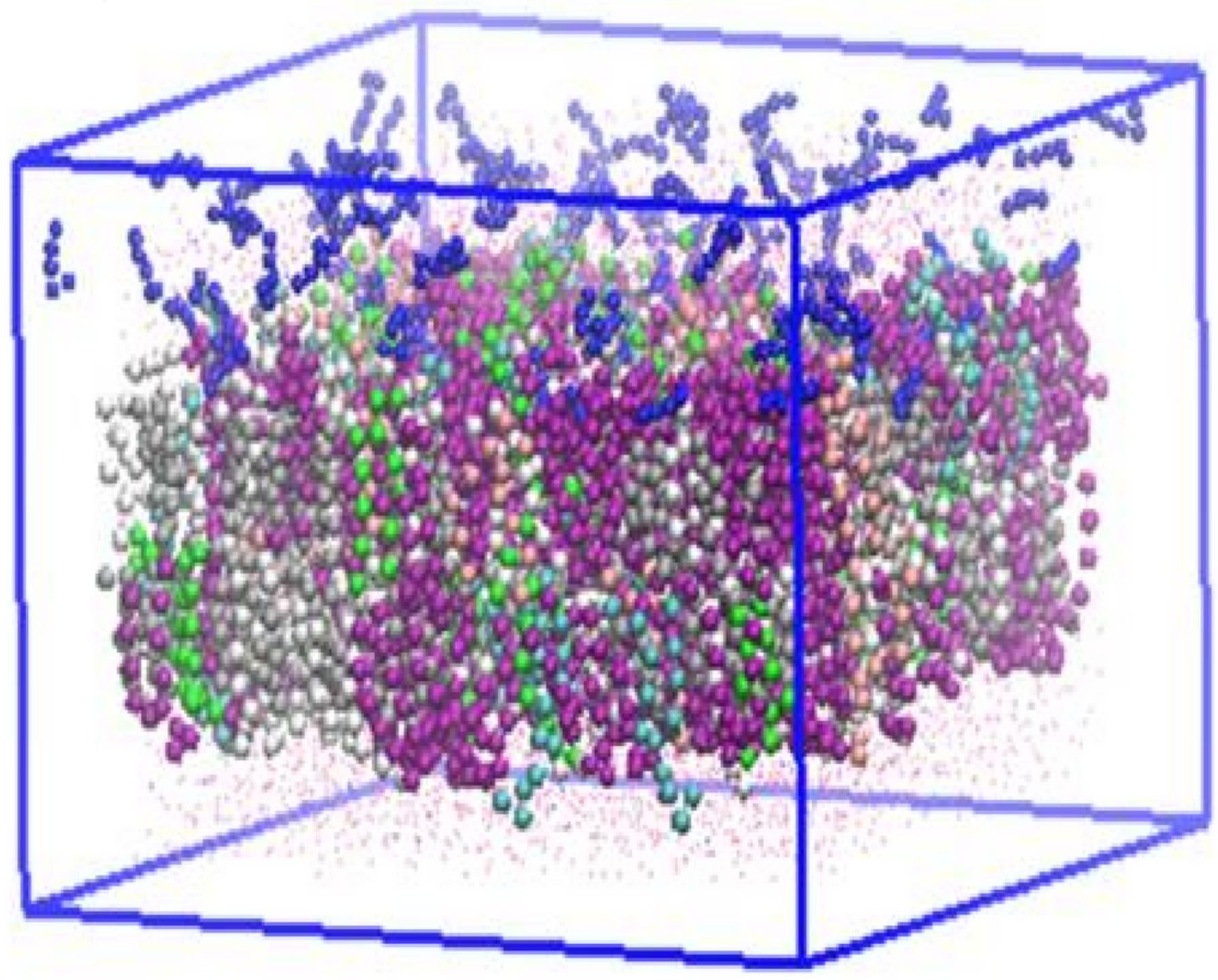

Figure 2

Schematic diagram of the free diffusion model. Blue beads represent drug molecules. Below them is a model of the small intestinal epithelial cells constructed in this paper 

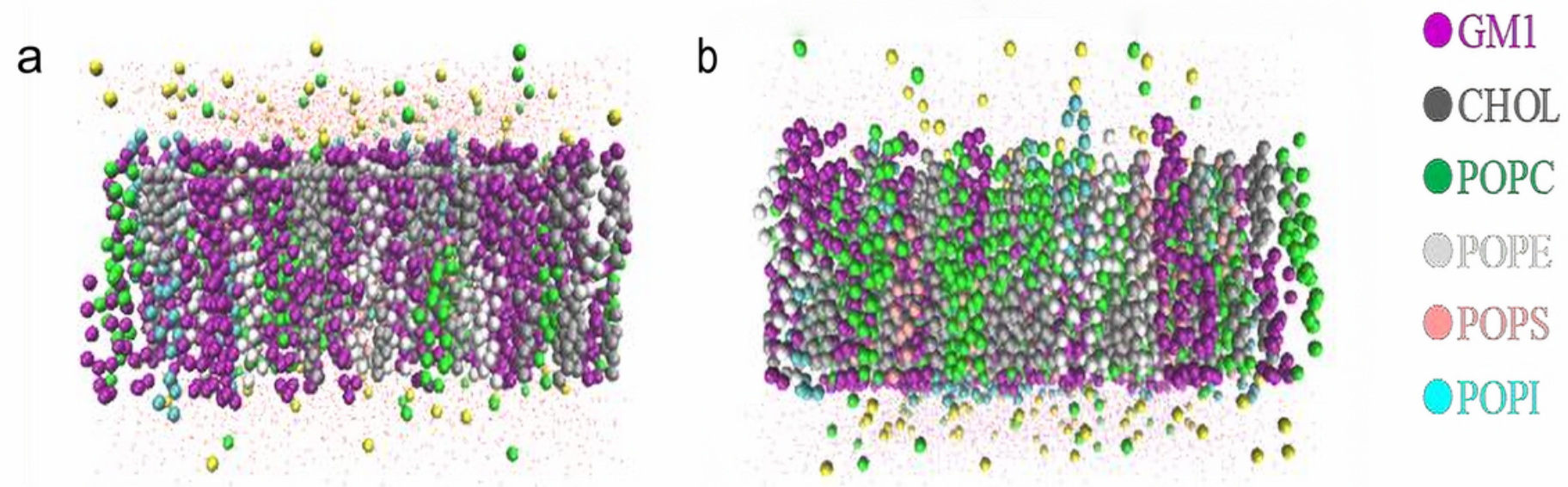

Figure 3

Visualization of the AP membrane (a) and BL membrane (b) in VMD
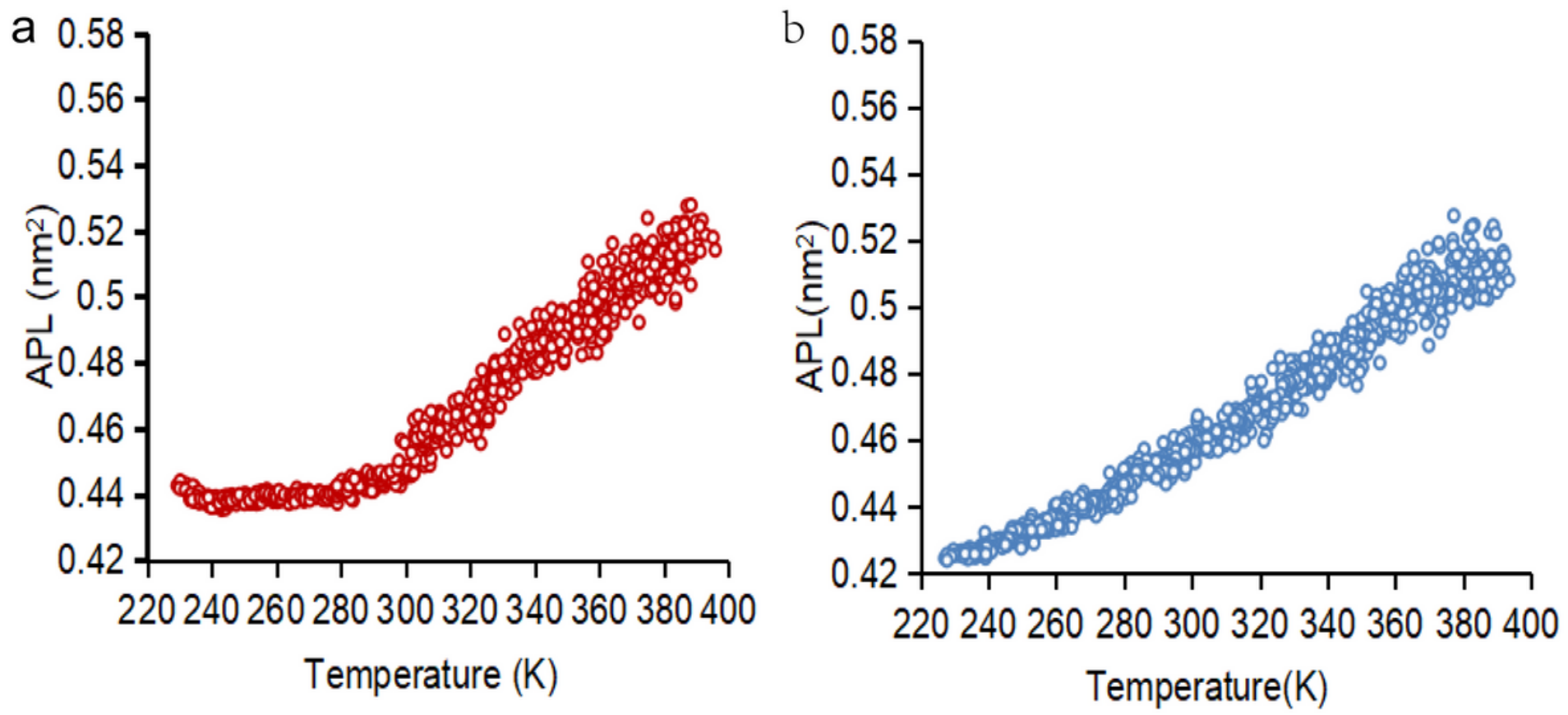

Figure 4

APL of AP with temperature during annealing simulation. $a$ : heating; $b$ : cooling. 

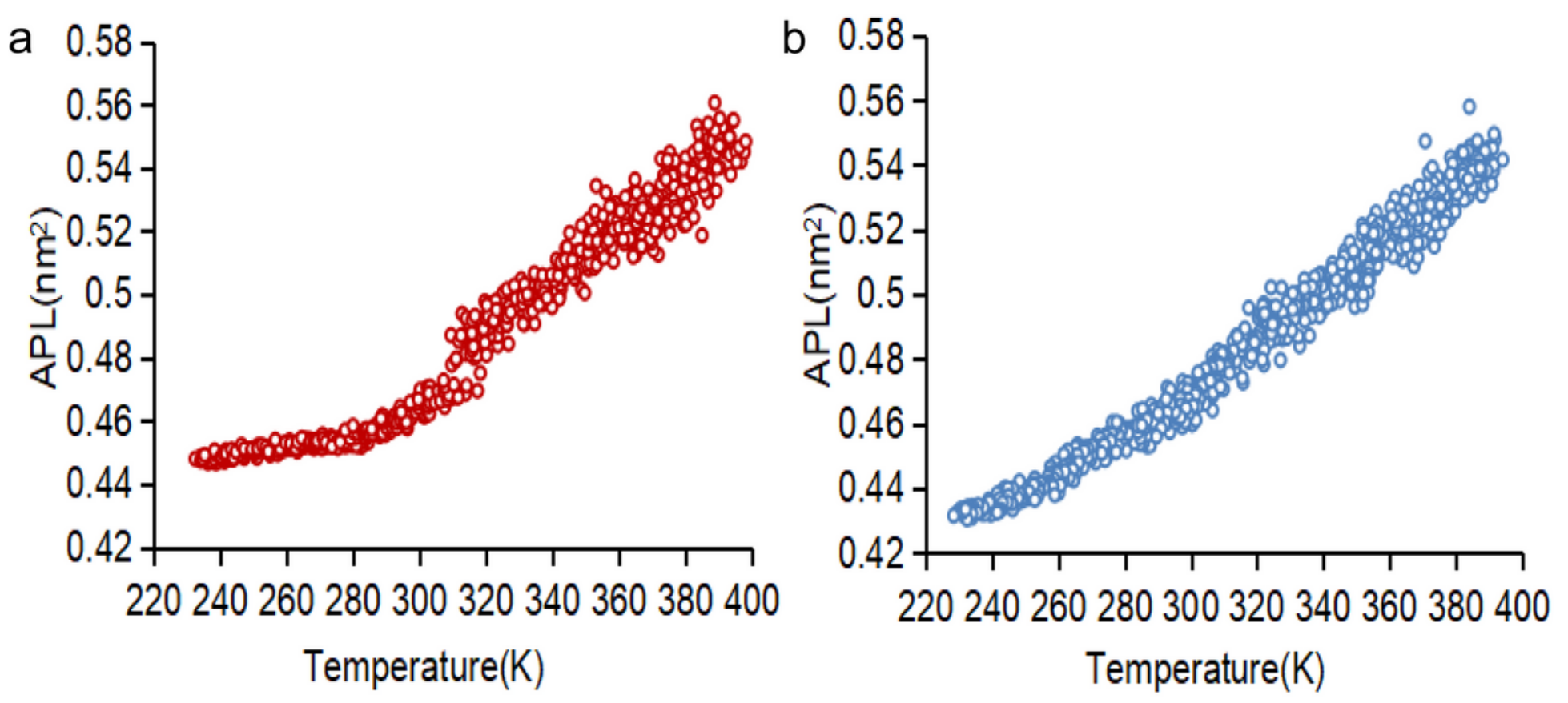

Figure 5

APL of BL with temperature during annealing simulation. $a$ : heating; $b$ : cooling.

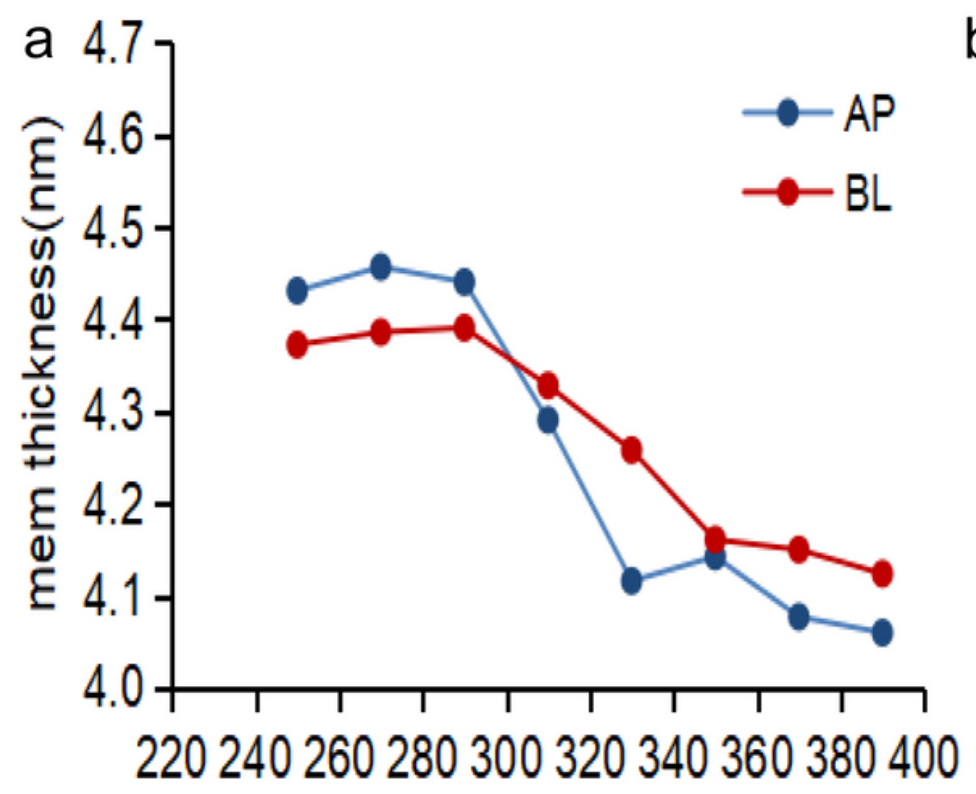

Temperature $(K)$

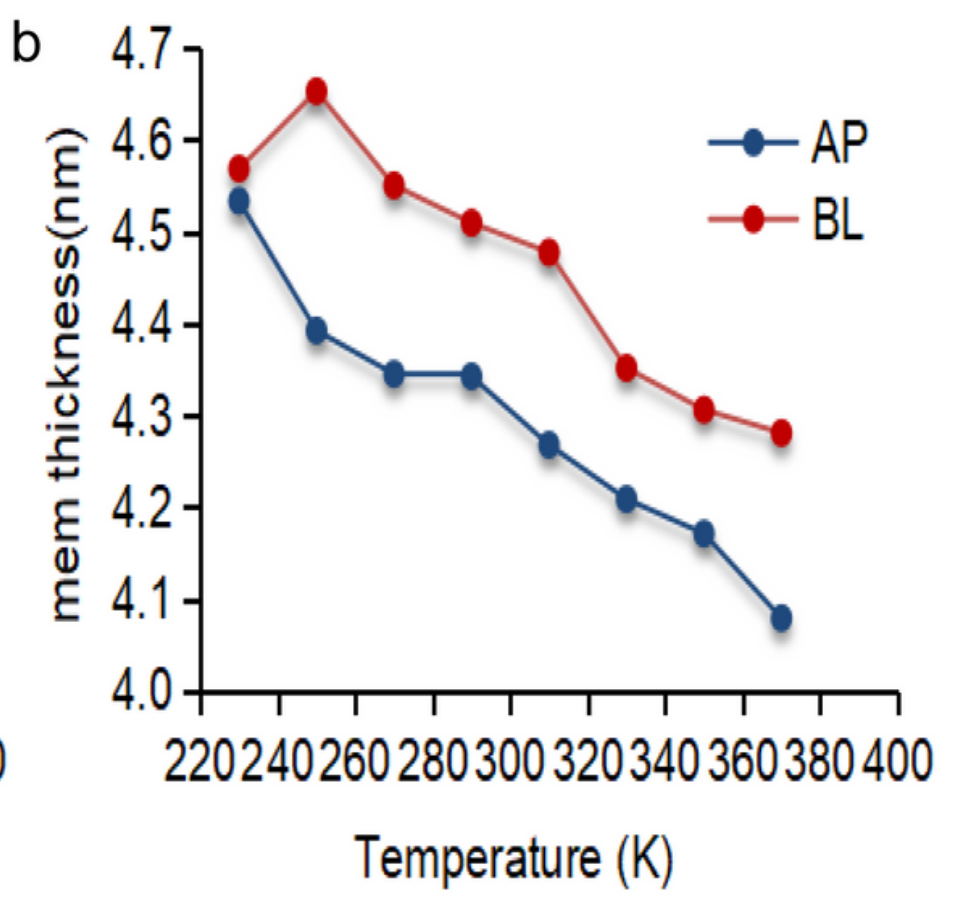


Figure 6

Variation of film thickness with temperature during annealing simulation. a: heating; b: cooling

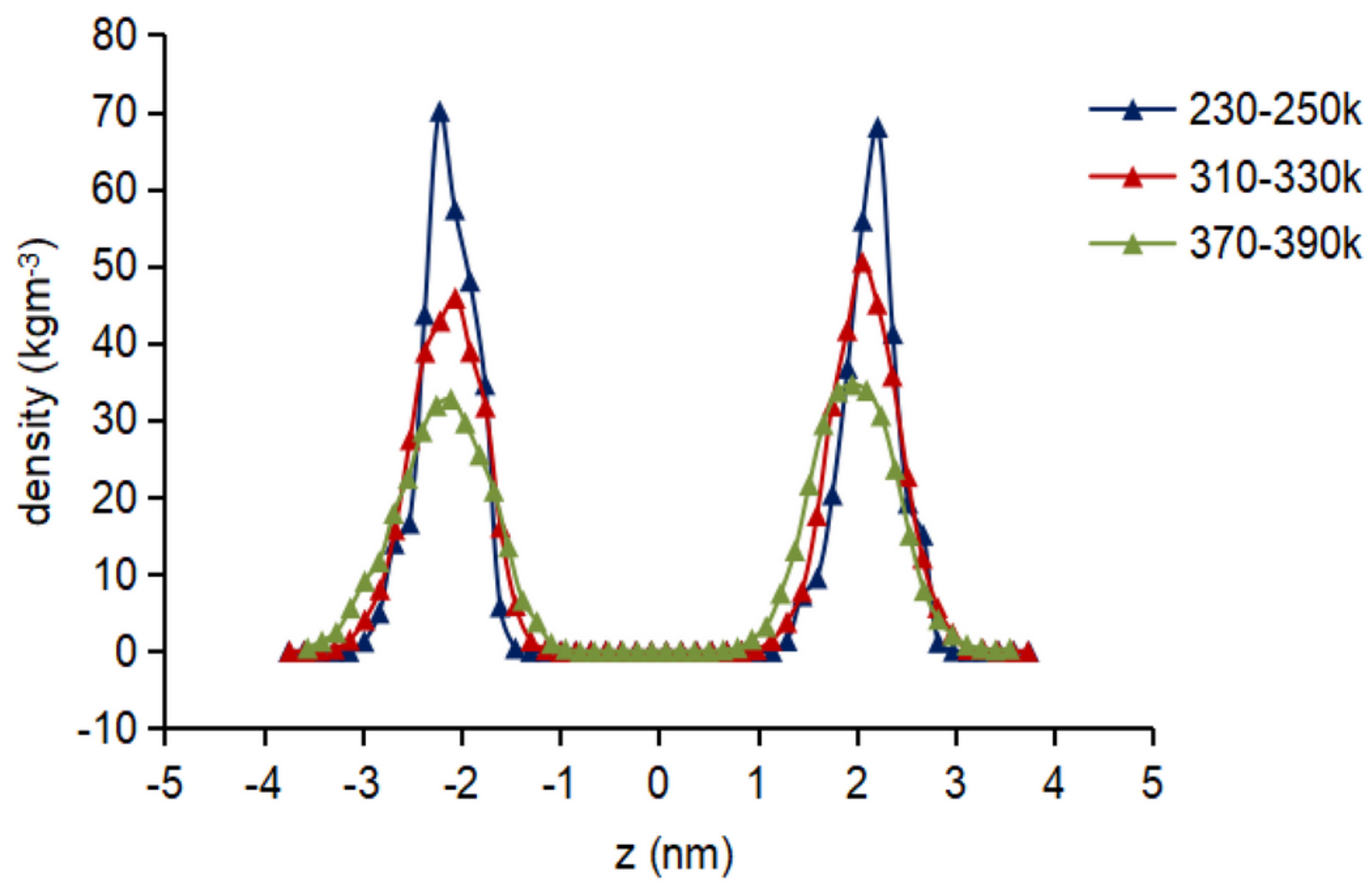

Figure 7

Density distribution diagram of lipid head beads along the Z-axis during the heating process of AP membrane. Black is $230 \mathrm{~K}-250 \mathrm{~K}$, red is $310 \mathrm{~K}-330 \mathrm{~K}$, and blue is $370 \mathrm{~K}-390 \mathrm{~K}$. 
a

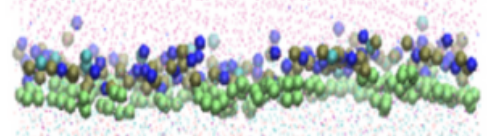

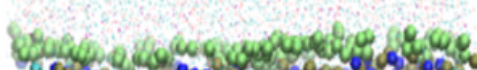
Thongen L

d
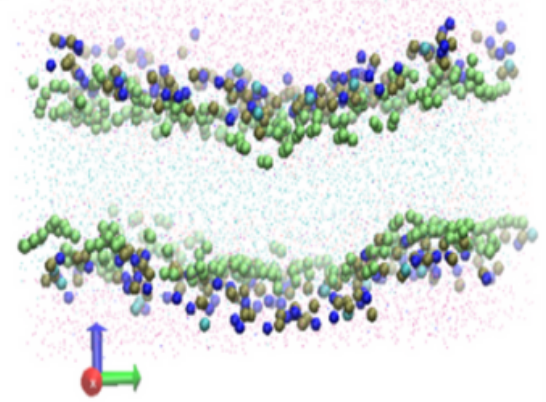

b
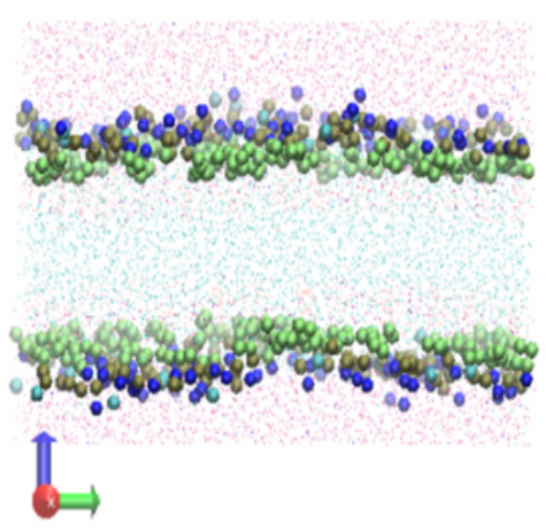

e

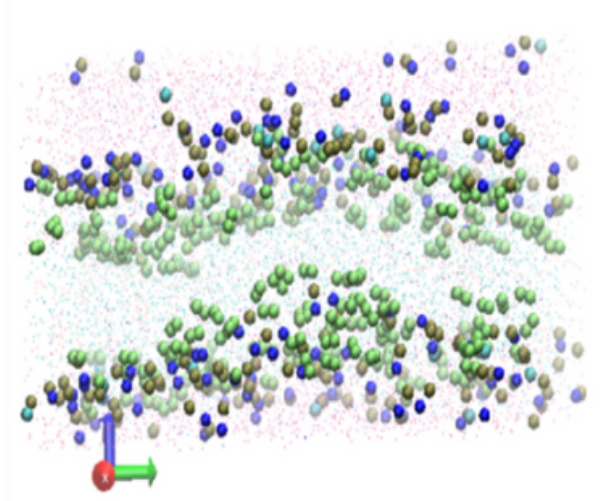

C
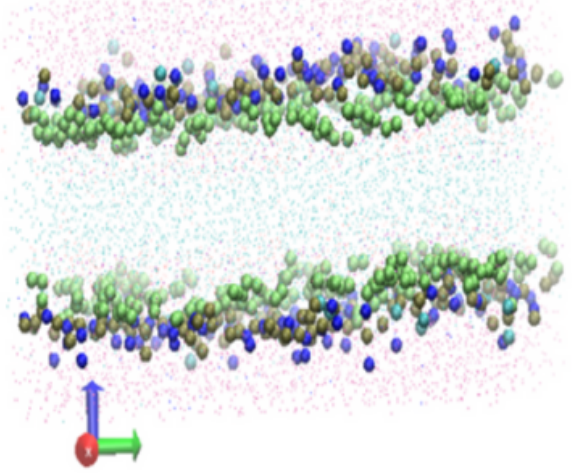

$\mathbf{f}$

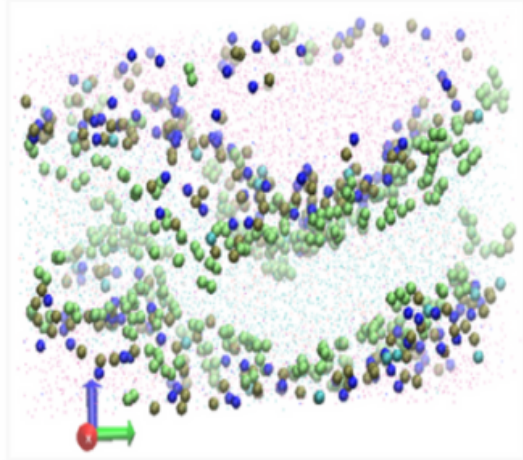

Figure 8

Lipid membrane morphology changes with temperature during AP heating (a:250K; b:280 K; c:310 K; d:340 e:370 K; f:390 K)
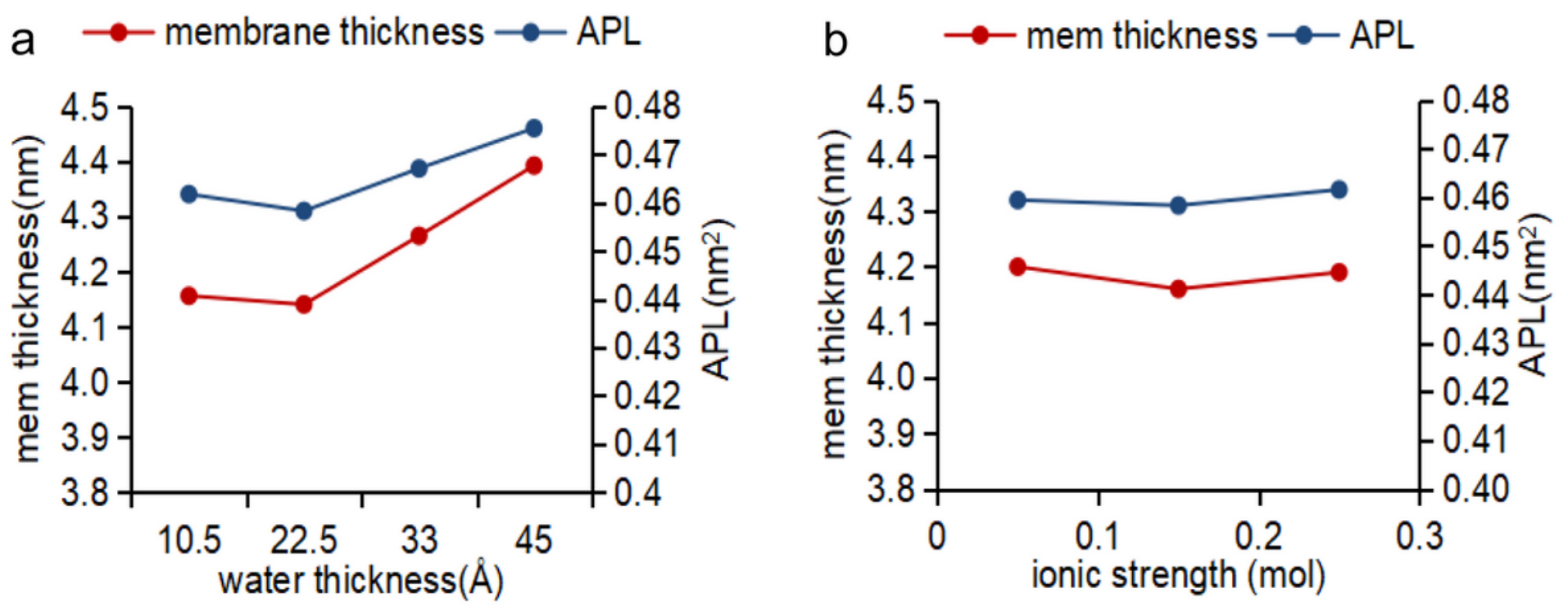

Figure 9

Effects of water layer thickness (left) and ionic strength (right) on membrane properties. Red represents film thickness, and blue represents APL. 

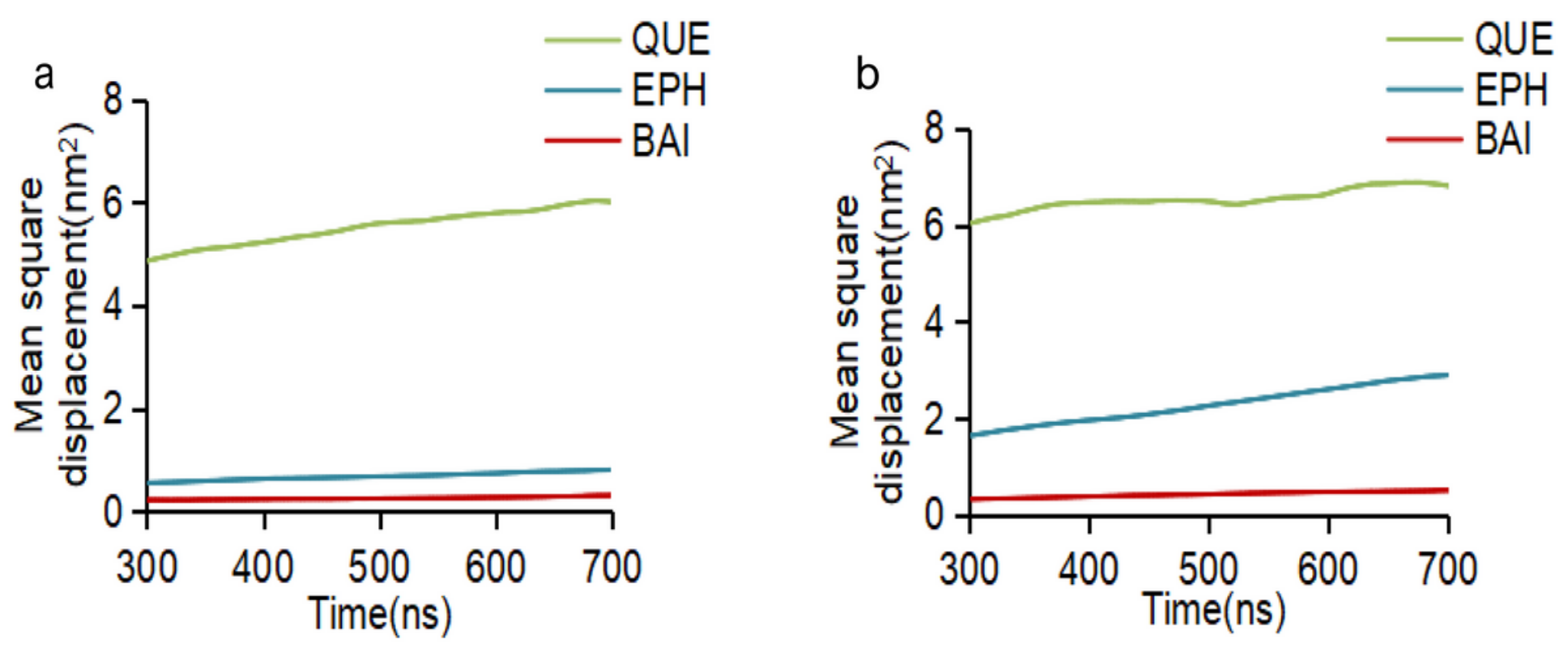

Figure 10

MSD of each drug in the z-axis direction of AP and BP membranes. A: AP-Z; B: BL-z. Red is BAl, blue is $\mathrm{EPH}$, and green is QUE.

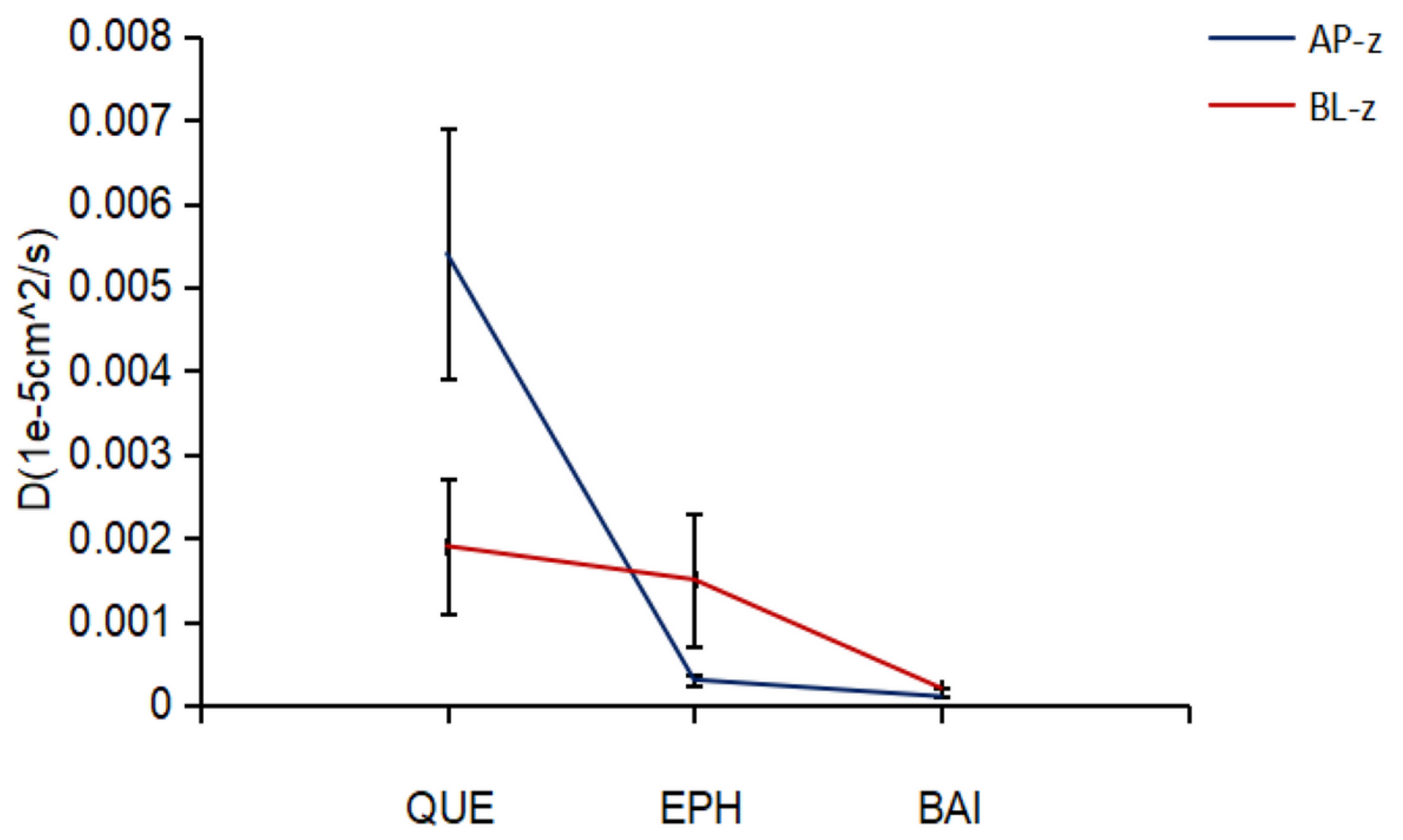


Figure 11

diffusion coefficient $D$ of each drug in the z-axis direction of AP and BP membranes. Red is BAl, blue is $\mathrm{EPH}$, and green is QUE.
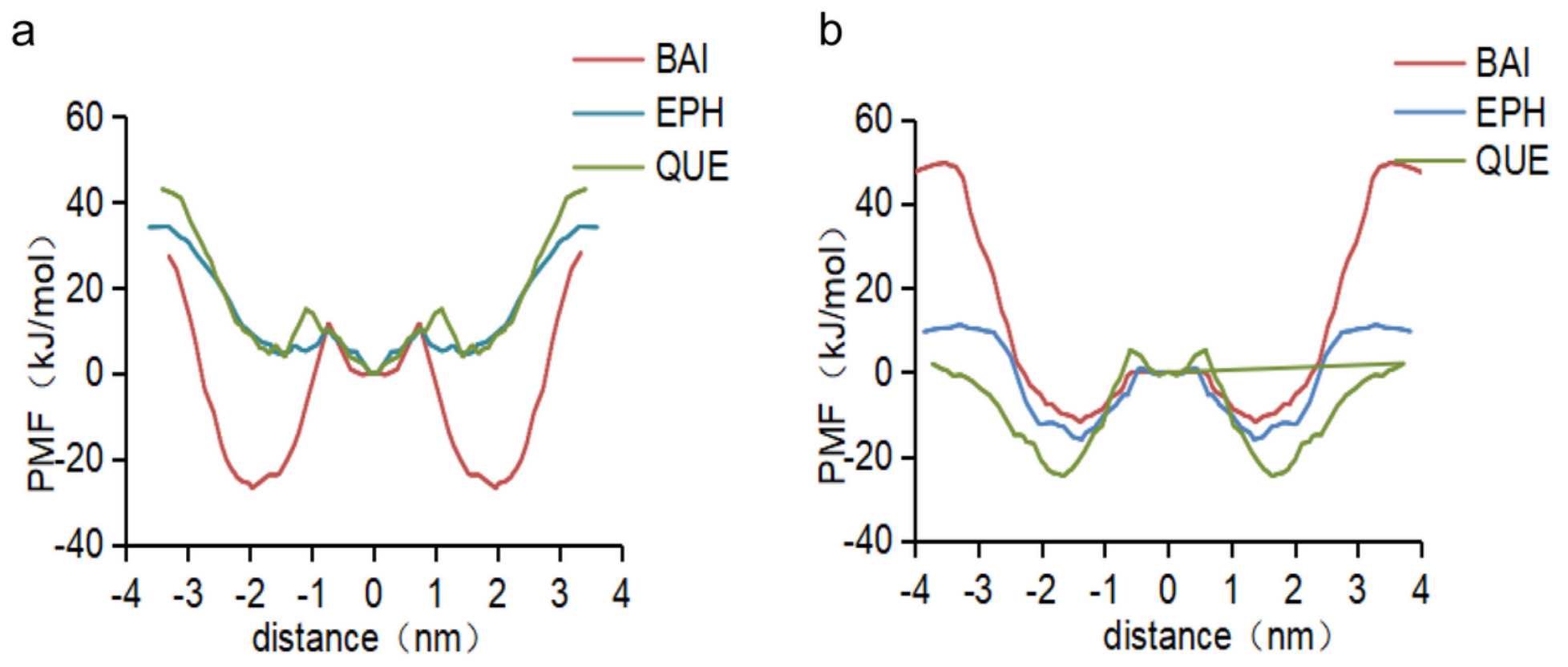

Figure 12

The PMF diagram of the z-axis. A: AP-Z; B: BL-z. Red is BAl, blue is EPH, and green is QUE.

\section{Supplementary Files}

This is a list of supplementary files associated with this preprint. Click to download.

- Abstractgraphic.tif 